Louisiana State University

LSU Digital Commons

$10-14-2014$

\title{
Measurement of the second order coherence of pseudo-thermal light in the azimuthal degree of freedom
}

\author{
Robert M. Cross \\ University of Rochester \\ Omar S. Magaña-Loaiza \\ University of Rochester Institute of Optics \\ Mohammad Mirhosseini \\ University of Rochester Institute of Optics \\ Robert W. Boyd \\ University of Rochester Institute of Optics
}

Follow this and additional works at: https://digitalcommons.Isu.edu/physics_astronomy_pubs

\section{Recommended Citation}

Cross, R., Magaña-Loaiza, O., Mirhosseini, M., \& Boyd, R. (2014). Measurement of the second order coherence of pseudo-thermal light in the azimuthal degree of freedom. Frontiers in Optics, FiO 2014 https://doi.org/10.1364/fio.2014.ftu1g.6 


\title{
Measurement of the Second Order Coherence of Pseudo-Thermal Light in the Azimuthal Degree of Freedom
}

\author{
Robert M. Cross ${ }^{1,}$, Omar S. Magaña-Loaiza², Mohammad Mirhosseini², Robert W. \\ Boyd $^{2,3}$ \\ ${ }^{1}$ Department of Physics \& Astronomy, University of Rochester, 206 Bausch \& Lomb Hall, Rochester, NY 14627, USA \\ ${ }^{2}$ The Institute of Optics, University of Rochester, 320 Wilmot Bldg, 275 Hutchison Road, Rochester, NY, 14627, USA \\ ${ }^{3}$ Department of Physics, University of Ottawa, Ottawa, Ontario K1N6N5, Canada \\ *rcross@pas.rochester.edu
}

\begin{abstract}
Using an angular version of the Hanbury Brown Twiss interferometer, we show for the first time second-order interference in the azimuthal degree of freedom, which exhibits higher resolution fringes than if coherent light were used.
\end{abstract}

(C) 2014 Optical Society of America

OCIS codes: $000.1600,260.3160$.

\section{Introduction}

In the last sixty years, light correlations have been a great subject of interest. The so called Hanbury Brown and Twiss (HBT) effect [1], one of the pioneering developments of quantum optics, has motivated a wide variety of research and fundamental questions regarding the nature of light. Besides HBT, many other experiments aiming to characterize correlations in different degrees of freedom involve a series of measurements of the second-order coherence function, $g^{(2)}$. Remarkable examples include the study of correlations of two photons in both the spatial and temporal domains $[2,3]$. Despite the interesting physics that light carrying orbital angular momentum involves, correlations of thermal light in the azimuthal degree of freedom have not yet been studied.

Previous works on classical correlations of thermal light used a rotating ground glass plate to mimic a source of thermal light [2]. We use a new technique to encode amplitude and phase information onto a fast digital micromirror device (DMD) [4]. This allow us to impart a laser beam with certain qualities of thermal light using Kolmogorov phase screens. Kolmogorov phase screens are used to simulate the distortion effects on wavefronts of light caused by propagation in turbid or random media. This model permits one to simulate a more realistic source of thermal light in a controlled and flexible manner. Our objective is the study of second-order interference of thermal light in the azimuthal degree of freedom.

\section{Experiment}

Our experimental setup can be considered to be an angular version of the HBT interferometer. As shown in Fig. 1, we first collimate a HeNe laser onto a DMD which rapidly displays pregenerated Kolmogorov screens. At the DMD,

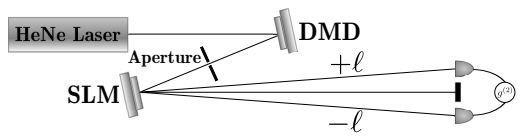

Fig. 1. Experimental setup

the beam gains a random phase component that is determined by Kolmogorov statistics. The beam is focused down, after which the first diffraction order is picked by an aperture. The beam illuminates a spatial light modulator (SLM) that performs two functions. We encode amplitude and phase information into a binary grating that is displayed on the SLM. The grating includes an angular double slit aperture multiplied by a forked hologram that projects the beam onto an orbital angular momentum component. The first positive and negative diffraction orders from the SLM are collected 
by single mode fibers and then integrated by two avalanche photodiode detectors that measure both the intensity in both arms and simultaneous events between the two arms.

For each value of $\ell$, we rapidly display thousands of realizations of Kolmogorov screens in order to simulate turbulent media. Summing over all screens, the first diffraction orders after the SLM can be described by

$$
E_{1}=\sum_{j} \alpha_{j, 1} e^{i \theta_{j, 1}}+\beta_{j, 1} e^{i \gamma_{j, 1}-i \ell \Delta \phi} \quad \text { and } \quad E_{2}=\sum_{j} \alpha_{j, 2} e^{i \theta_{j, 2}}+\beta_{j, 2} e^{i \gamma_{j, 2}+i \ell \Delta \phi},
$$

where $E_{1}$ and $E_{2}$ represent the field in the first positive and negative diffraction orders, respectively. The terms $\alpha_{j} e^{i \theta_{j}}$ and $\beta_{j} e^{i \gamma_{j}}$ represent the $j$ th realization of a Kolmogorov phase screen projected over the angular slits. The spatial coherence of the pseudo-thermal light can then be described by

$$
\int_{0}^{2 \pi} f(\phi) \delta_{j}(\phi) e^{-i \ell \phi} d \phi=\alpha_{j} e^{i \theta_{j}} \quad \text { and } \quad e^{-i \ell \Delta \phi} \int_{0}^{2 \pi} f(\phi) \delta_{j}(\phi+\Delta \phi) e^{-i \ell \phi} d \phi=\beta_{j} e^{i \gamma_{j}} e^{-i \ell \Delta \phi},
$$

where $f(\phi)$ represents the transmission of the angular slits and $\delta(\phi)$ represents a Kolmogorov phase screen, using $\phi$ as the azimuthal coordinate. We can then calculate the second-order correlation function,

$$
g^{(2)}=\left\langle I_{1} I_{2}\right\rangle-\left\langle I_{1}\right\rangle\left\langle I_{2}\right\rangle
$$

After combining these expressions, we can compute the second-order correlation function to be

$$
g^{(2)}=2 \Re\left(\left\langle\alpha_{j, 1} \beta_{j, 1}^{*} \beta_{j, 2} \alpha_{j, 2}^{*} e^{i\left(\theta_{j, 1}-\theta_{j, 2}+\gamma_{j, 1}-\gamma_{j, 2}\right)}\right\rangle\right) \cos (2 \ell \Delta \phi) .
$$

Remarkably, interference fringes with increased resolution are formed, although the visibility is decreased.

\section{Results}

After choosing the size of the angular slits to be $15^{\circ}$, and the angular slit separation to be $30^{\circ}$. The experiment was then run with and without the turbulent Kolmogorov screens. Measurements were taken for projections of $\ell=0$ to 16 in the first arm (and correspondingly, $\ell=0$ to -16 in the second arm). For the measurements that include turbulence, the DMD cycled through 72000 pregenerated Kolmogorov screens at a rate of $1440 \mathrm{~Hz}$. The experimental results in Fig. 2 show that using a coherent beam gives first-order interference (FOI) fringes that modulate as $\cos (\ell \Delta \phi)$ in both the singles and $g^{(2)}$ correlation measurements. As soon as we turn on the Kolmogorov screens, we can see that the interference fringes show increased resolution, as the $g^{(2)}$ correlation measurements modulate at $\cos (2 \ell \Delta \phi)$.
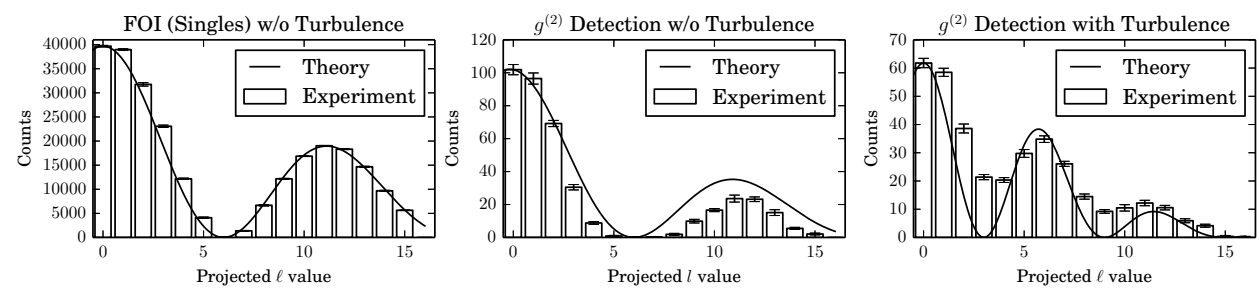

Fig. 2. Experimental results of first-order interference and $g^{(2)}$ measurements with and without turbulence. Note that the interference fringes in the turbulent $g^{(2)}$ measurements modulate twice as much.

\section{References}

1. R. H. Brown and R. Q. Twiss, "LXXIV. A new type of interferometer for use in radio astronomy," Philosophical Magazine Series 7 45, 663-682 (1954).

2. G. Scarcelli, V. Berardi, and Y. Shih, "Can Two-Photon Correlation of Chaotic Light Be Considered as Correlation of Intensity Fluctuations?” Phys. Rev. Lett. 96, 63,602 (2006).

3. A. Nevet, A. Hayat, P. Ginzburg, and M. Orenstein, "Indistinguishable Photon Pairs from Independent True Chaotic Sources,” Phys. Rev. Lett. 107, 253,601 (2011).

4. M. Mirhosseini, O. S. Magaña Loaiza, C. Chen, B. Rodenburg, M. Malik, and R. W. Boyd, "Rapid generation of light beams carrying orbital angular momentum,” Opt. Express 21, 30,196-30,203 (2013). 